\title{
INVESTIGACIONES
}

\section{Estudio metodológico de las variables que componen el constructo de "apoyo y participación de la familia en los procesos educativos"}

\author{
Methodological study of the variables involved in the construct of "support and \\ participation of the family in educational processes"
}

\author{
Carlos Miranda Carvajal ${ }^{a}$, Pablo Castillo Armijo ${ }^{b}$ \\ ${ }^{\text {a }}$ Facultad de Ciencias de la Educación, Universidad de Talca. \\ Correo electrónico: cmc.mira@gmail.com \\ ${ }^{\text {b}}$ Facultad de Ciencias de la Educación, Universidad de Talca. \\ Correo electrónico: pacastillo@utalca.cl.
}

\begin{abstract}
RESUMEN
Estudio metodológico sobre los conceptos teóricos "comunicación con la escuela", "apoyo y supervisión del aprendizaje en el hogar" y "conocimiento de la escuela" que componen el constructo "participación y apoyo familiar en los procesos educativos" en una muestra de apoderados de $6^{\circ}$ año básico pertenecientes a 6 escuelas de dependencia municipal en la comuna de Calama, Chile. Consideró la implementación de una metodología correspondiente al enfoque cuantitativo por medio de un análisis factorial de carácter confirmatorio ( $\mathrm{AFc}$ ). El análisis de los 32 ítems que conforman el instrumento elaborado y aplicado, permite afirmar, substantiva y significativamente, residuos redundantes mayores que $60 \%(\mathrm{p}<0,05)$, y la validez constructiva de los mismos.

Palabras claves: participación y apoyo familiar/comunicación con la escuela/apoyo y supervisión del aprendizaje en el hogar/conocimiento de la escuela.
\end{abstract}

\section{ABSTRACT}

This methodological study explores theoretical concepts including "communication with the school," "support and supervision of learning at home," and "knowledge of the school," which comprise the construct "family support and involvement in educational processes." The sample was taken from parents whose children study at six municipal schools in the city of Calama. For the purpose of this study, a quantitative methodology was used through a confirmatory factor analysis (CFA). The results of the analysis of the 32 items that comprise the instrument that was designed and applied suggest redundancy features above $60 \%(\mathrm{p}<0.05)$ and point to the validity of the construct.

Keywords: family support and involvement, communication with school, support and learning supervision at home, knowledge of the school. 
Estudios Pedagógicos XLIV, N $^{\circ}$ 1: 115-133, 2018

ESTUDIO METODOLÓGICO DE LAS VARIABLES QUE COMPONEN EL CONSTRUCTO DE "APOYO Y PARTICIPACIÓN DE LA FAMILIA EN LOS PROCESOS EDUCATIVOS"

\section{INTRODUCCIÓN}

El principal propósito para introducirse en los conceptos que componen la comunidad educativa y tener una mirada sobre los factores que influyen en la mejora o que son pilares de los procesos de enseñanza y aprendizaje, se genera a partir de la revisión de la literatura especializada. Es en ella donde se ponen a la vista un abanico de posibilidades donde potenciar elementos tales como liderazgo, las altas expectativas y metas educativas claras entre otros, que pueden determinar los logros académicos de carácter cualitativo y cuantitativo.

La investigación nos da cuenta de diferentes análisis y miradas que reflejan una o varias caracterizaciones del funcionamiento de los factores asociados al aprendizaje escolar, y las variables que los componen en la búsqueda de mejores aprendizajes (Cornejo y Redondo 2007).

Variados estudios muestran estos factores en un funcionamiento cooperativo, donde todos colaboran con un objetivo, la mejora de los procesos de enseñanza y aprendizaje, derivados en una optimización de los resultados de la evaluación interna y externa de la institución educativa (Álvarez, Aguirre y Vaca 2010; Anabalón, Carrasco, Díaz, Gallardo y Cárcamo 2008; Arancibia 1992; Bellei, Valenzuela, Vanni y Contreras 2014; Belmar 2003; Bolívar 2006; Brunner y Elacqua 2004; Cano y Casado 2015; Coraggio 1997; Cornejo y Redondo 2007; Epstein y Sheldon 2008; Fullan 2011; Gerstenfeld 1995; Himmel, Maltes y Majluf 1984; Jadue 1999, 2003; LLECE/UNESCO 2002; Murillo 2007; Pizarro y Clark 1987; Pizarro 2009, 2012; Ruiz 2003; UNESCO 2008, 2012, 2014, 2015; UNICEF 2004; Valdés, Carlos y Arreola 2013; Valdés, Martin y Sánchez 2009; Vera, González y Hernández 2014; Yurén y Cruz 2009; Zárate 1992).

Los factores asociados al mejoramiento en educación de la eficacia escolar son variados. Por lo general obedecen a los contextos en los que se encuentra emplazada la institución educativa por lo que no todos pueden ser observados desde el mismo punto de vista. Tampoco ser replicados de la misma forma de un contexto a otro, e incluso ser medidos de manera objetiva. Es necesario mencionar que existen puntos comunes y que en algunos casos poseen una misma naturaleza y por tanto pueden ser observados y medidos de manera más objetiva, uno de estos factores es la participación de la familia en los procesos educativos.

Fullan propone que, en la búsqueda de la innovación educativa para mejorar los sistemas, es necesario realizar un trabajo más profundo sobre los puntos comunes que permiten esta mejora, dando especificidad y precisión al interiorizarse en prácticas particulares en la mejora e innovación en educación (Fullan 2011).

De esta manera es que las propuestas de Fullan junto con la de Cornejo y Redondo cobran sentido al poder tomar cada una de las variables, sea el liderazgo, las expectativas del profesor, participación familiar u otra que se proponga como clave de los procesos de funcionamiento de las escuelas efectivas, ahondando en su naturaleza y poniendo a la vista su real aporte a los logros de resultados en los aprendizajes de los estudiantes (Fullan 2011; Cornejo y Redondo 2007).

El alcance propuesto para la investigación es confirmatorio, ya que se investiga un constructo teórico conocido desde la investigación (Arancibia 1992; Bellei et al. 2014; Bellei, Morawietz, Valenzuela y Vanni 2015; Brunner y Elacqua 2004; Cornejo y Redondo 2007; Epstein y Sheldon 2008; Gerstenfeld 1995; Himmel et al. 1984; LLECE/UNESCO 2002; Murillo 2007; Ruiz 2003; UNICEF 2004; UNESCO 2008; Valdés et al. 2009; Valdés 
et al. 2013; Yurén y Cruz 2009; Zárate 1992). Caracterizado desde lo cuantitativo en trabajos del constructo currículum del hogar y currículum del hogar mejorado (Pizarro y Clark 1987; Pizarro 2009, 2012).

La investigación permite hacer una validación de los ítems del instrumento creado para medir el apoyo y participación de la familia en los procesos educativos, y determinar ¿cómo se reflejan los ítems respecto a la teoría y conceptos usados para definir específicamente el rol de la comunicación con la escuela, el apoyo y supervisión del aprendizaje en el hogar y el conocimiento de la escuela?, considerando que el instrumento posee en su concepción tres variables con características comunes e independientes entre sí.

\section{MARCO TEÓRICO REFERENCIAL}

La composición del apoyo familiar en las labores educativas se expresa claramente en las definidas como variables de la escuela y variables de origen de los estudiantes. Estas últimas se clasifican en variables de la comunidad de origen, variables del hogar de origen (Coraggio 1997; Cornejo y Redondo 2007; Gerstenfeld 1995), y componentes del currículum del hogar (Pizarro y Clark 1998; Pizarro 2009).

Propuesto desde la visión de Brunner y Elacqua las dos grandes variables poseen influencias en los aprendizajes de los estudiantes, las que pueden ser positivas o negativas. La escuela posee mayormente influencias positivas, sin embargo, la variable de origen y su composición posee un igual porcentaje de influencias positivas y negativas en los logros escolares (Brunner y Elacqua 2004).

Es necesario considerar como factor determinante a la participación familiar en los procesos educativos visualizando los beneficios que aporta esta relación a los logros, sean estos destacados o descendidos en la escuela (Álvarez et al. 2010).

Por su parte, la investigación en América Latina aporta con evidencia en relación a las expectativas de los padres sobre el desempeño de sus hijos: la relación entre altas expectativas y un mejor desempeño académico de los estudiantes no está garantizada, pero se observa que existe una asociación significativa (Bazán, Sánchez y Castañeda 2007).

Mediante avanza la investigación sobre cómo realmente se incluye a la familia en los procesos de enseñanza y aprendizaje, también se pueden encontrar opiniones que difieren de su importancia. Principalmente en la opinión de profesores en formación que consideran a la familia sólo desde el punto de vista de la vulnerabilidad social y tienden a resaltar los aspectos negativos de éstas, llegando a ignorar las capacidades que pueden ofrecer para el logro de un entorno educativo apropiado (Gomila y Pascual 2015).

Existen autores que van más allá de relacionar el factor de apoyo familiar con la adquisición de aprendizajes efectivos, sino que la sitúan en un ámbito de inclusión mayor que puede alterar varios otros procesos de mejoramiento de la escuela (Arancibia 1992; Bellei et al. 2014, 2015; Himmel et al. 1984; LLECE/UNESCO 2002; Murillo 2007; UNESCO 2008, UNICEF 2004; Zárate 1992).

Una indiscutible participación familiar en la escuela se torna en el núcleo del desarrollo de los procesos educativos, según Anabalón et al. (Anabalón et al. 2008) se compone de distintas variables que se desarrollan y concretizan desde distintos ámbitos de participación (Epstein y Sheldon 2008; Ruiz 2003; Pizarro 2006, 2009; Valdés et al. 2009; Valdés et al. 2013; Yurén y Cruz 2009). 
Además, observar variables que componen la participación de la familia en la comunidad escolar como la crianza, colaboración con la comunidad, toma de decisiones voluntariado, comunicación con la escuela (Epstein y Sheldon 2008); junto con Valdés et al. (Valdés et al 2009), fomento al cumplimiento de metas (Ruiz 2003); influencia en la deserción escolar (Yurén y Cruz 2009), y la ayuda y apoyo en las tareas escolares (Epstein y Sheldon 2008; Pizarro 2006, 2009; Ruiz 2003; Yurén y Cruz 2009).

Poniendo en análisis las variables antes mencionadas, existe la propuesta de tres grandes dimensiones que engloben y mantengan estables las variables de estudios anteriores: a) Comunicación con la escuela y práctica de los padres para mantener la comunicación e intercambio de información con los profesores sobre el aprendizaje de los estudiantes. b) Apoyo del aprendizaje en el hogar y prácticas que promueven la comunicación sobre el aprendizaje entre familia y estudiantes. c) Conocimiento de la escuela y sus generalidades de funcionamiento (Valdés et al. 2013).

Encontramos que una de las variables descriptoras como factor asociado que influye directamente en el aprendizaje escolar es el apoyo y supervisión del aprendizaje en el hogar. Según algunos autores la familia actúa como mediador de este aprendizaje por medio de una guía y apoyo académico en el hogar (Álvarez et al. 2010, Anabalón et al. 2008; Belmar 2003; Bolívar 2006; Cano y Casadol 2015; Cornejo y Redondo 2007; Epstein y Sheldon 2008; Jadue 1999, 2003; Pizarro 2006, 2009; Ruiz 2003; UNESCO 2012, 2014 y 2015; Valdés et al. 2009; Valdés et al. 2013; Vera et al. 2014).

En la propuesta de observar a la escuela como una comunidad que permite el desarrollo integral de los estudiantes, es decir desarrollando las áreas intelectuales y afectivas en igual importancia, el factor familia poseerá el mayor impacto en la mejora de estos procesos (Belmar 2003).

La consideración que la educación es un proceso individual, pero con un enorme impacto en la construcción social del individuo (Belmar 2003). Se transforma en la base de una propuesta donde el uso de las herramientas y materiales educativos por parte de la familia, es decir su involucramiento directo como corresponsable del aprendizaje de sus pupilos contribuye a realzar los resultados de aprendizaje de los mismos (UNESCO 2014).

La influencia de la familia en los procesos educativos también se puede entender como la relación de los procesos familiares, como transformación de la cultura contemporánea, donde a pesar de los cambios en los estilos de vida de la sociedad, la estructura familiar sigue determinando objetivos y metas sociales como educativas (Jadue 2003).

La familia ejerce una poderosa influencia en el proceso de enseñanza y aprendizaje por medio del apoyo, la supervisión y la transformación de los componentes de la familia en mediadores y colaboradores de los procesos educativos. Influye positivamente en la realización de trabajos escolares, mejoras en las percepciones de la evaluación y la apropiación de patrones disciplinarios dentro de la escuela (Bazán et al. 2007; Jadue 2003).

En un trabajo con niños chilenos, encontraron que la variable nivel socioeconómico tiene poco poder explicativo en el desempeño académico de los estudiantes, mientras que la alta expectativa de la madre sobre la carrera académica de sus hijos y el nivel de escolaridad del padre explican mejor el desempeño de los estudiantes (Bazán et al. 2007).

Es en este punto donde la participación de las familias en la escuela chilena se comienza a evidenciar de una manera potente. El interés que poseen los padres sobre la educación de sus hijos indica una prolongación de las expectativas y aspiraciones sociales de las familias. Estas relacionan directamente los componentes académicos y sociales de una comunicación 
familia-escuela en las cuales las exigencias de altos resultados académicos evidencian en un futuro un crecimiento en estatus socioeconómico desde la educación (Jadue 1999; Villarroel 2002; Bruner 2003; UNICEF 2004; Bellei et al. 2014, 2015; UNESCO 2015).

Existen propuestas, como la de Álvarez et al, quien coincide con Epstein y Sheldon, en que la creación y descentralización de las ideas, tanto de la familia como de la escuela sobre el logro de mejoras en los procesos educativos y la relaciones de estas "esferas de influencia", permiten mayor participación por la apertura de los centros educativos (Álvarez et al. 2010; Epstein y Sheldon 2008).

Yurén y Cruz (2009) señalan que la propuesta de apoyo y supervisión del aprendizaje en el hogar tiene como referente a la afirmación de que el apoyo que brindan los padres a los hijos en relación a las tareas, juega un papel primordial en la relación familia-escuela (Yurén y Cruz 2009). Este apoyo puede estar determinado por el valor que otorgan los padres a los aprendizajes escolares y su auto valoración en relación con éstos, independiente del nivel socio económico al que pertenezca la familia (Jadue 1999).

La UNESCO y UNICEF catalogan a los factores sociales y económicos como importantes, al igual que las políticas internacionales que se orientan a un mayor desarrollo en estos ámbitos y a la contribución de la educación a estas metas. Asimismo, al analizar los factores que influyen en la educación como práctica particular y visualizando a la escuela como una comunidad de aprendizaje, se observa principalmente en niveles sociales vulnerables que el apoyo y la supervisión de los procesos educativos influyen positivamente en los logros de los estudiantes, donde los componentes de este apoyo, las características de las familias y de los estudiantes en particular tienen una fuerte asociación con los logros de aprendizajes y con las expectativas educativas de los padres con relación a sus hijos, la revisión y comentarios sobre las calificaciones alcanzadas por los estudiantes. Sobre todo, la práctica de desarrollo de tareas en el hogar, en donde se afirma que los estudiantes que poseen apoyo familiar por lo menos 30 minutos diarios para esta práctica, alcanzan mejores resultados que quienes no (UNESCO 2000, 2008, 2011, 2014; UNICEF 2004).

La situación del estudiante en estas interacciones es central, ya que es considerado como el centro del proceso educativo. Por lo que la búsqueda de una educación de calidad se relacionaría directamente con el mayor o menor dinamismo y responsabilidad del rol de la familia dentro de la escuela (Cano y Casado 2015).

Esta relación educativa entre familia-escuela, como factor explicativo de una positiva influencia social en la mejora de los aprendizajes es de suma importancia de establecer (Vera et al. 2014).

Pizarro y Clark y Pizarro, al usar la estrategia educativa denominada currículum del hogar, han demostrado que su tamaño de error Delta Glass ha sido +0,75 sigmas; y con fuerte ganancia en guía y apoyo académico y presión académica. En contraste con las rutinas y modelos de lenguaje, que cambian muy poco a nivel de intervención, en todo caso, el modelo de Escuelas Efectivas predice entre 2-5 veces más que el modelo sociológico los aprendizajes formales de los estudiantes (Pizarro y Clark 1998; Pizarro 2008).

\section{MARCO METODOLÓGICO}

Para el estudio se consideró como variable dependiente al Apoyo y Participación Familiar en los Procesos Educativo; representado en las propuestas bibliográficas como un constructo 
que según los autores posee entre 3 y 8 variables (Álvarez et al. 2010; Anabalón et al. 2008; Arancibia 1992; Bellei et al. 2014, 2015; Epstein y Sheldon 2008; Gomila y Pascual 2015; Himmel et al. 1984; LLECE/UNESCO 2002; Murillo 2007; Ruiz 2003; UNESCO 2008; UNICEF 2004; Valdés et al. 2009; Yurén y Cruz 2009; Zárate 1992).

Como variables independientes se consideraron las dimensiones de "Comunicación con la Escuela", "Apoyo y Supervisión del Aprendizaje en el Hogar" y "Conocimiento de la Escuela", pertenecientes al constructo de Participación Familiar en los Procesos Educativos, aquí se consideraron 3 dimensiones planteadas como componentes de esta variable, extraídas de las propuestas de Epstein y Sheldon y Valdés et al., quienes proponen 8 y 3 variables, respectivamente (Epstein y Sheldon 2008; Valdés et al. 2009).

El diseño del presente estudio corresponde al tipo Cuantitativo "no experimental", transeccional o transversal de carácter confirmatorio metodológico-métrico. Existe análisis multivariado de las variables (se consideraran 3 factores en el instrumento aplicado), el que posee una naturaleza descriptiva de los factores presentes dentro de un constructo específico (Kerlinger y Lee 2002; Pizarro 2012).

"Comunicación con la Escuela" medido en 12 ítems con un puntaje máximo de 48 puntos, "Apoyo y Supervisión del Aprendizaje en el Hogar" medido en 12 ítems con un puntaje máximo de 48 puntos y "Conocimiento de la Escuela" medido en 8 ítems con un puntaje total de 32 ítems.

Los cuestionarios para padres y apoderados entregados, correspondió a una muestra inicial de 812 cuestionario, lo que se representa de la siguiente manera: Población $=1.468$ apoderados; Muestra Seleccionada $=898$ apoderados; Muestra Real $=812$ apoderados. La muestra correspondió al 61,38\% de la población. Para la muestra real entregada a los directores de los establecimientos educacionales, $\mathrm{N}=55,31 \%$ de la población, la muestra final con la que se desarrolló el estudio fue de $\mathrm{N}=520$ apoderados. Los instrumentos recuperados representan unos $68,71 \%$ de la muestra real de los test entregados, por lo que equivale a un $38,14 \%$ de la población.

La creación del cuestionario se desarrolló considerando el apoyo y participación de la familia en los procesos educativos, definido como un constructo que posee distintas variables. Epstein y Sheldon proponen 6 dimensiones a saber: crianza, comunicación con la escuela, voluntariado, supervisión del aprendizaje en casa, toma de decisiones y colaboración con la comunidad (Epstein y Sheldon 2008). Por su parte, Valdés et al. propone la existencia de dos variables a saber: comunicación con la escuela y apoyo del aprendizaje en casa (Valdés et al 2013). Pizarro y Clark en sus trabajos también muestran la presencia de la variable apoyo y supervisión del aprendizaje en el hogar (Pizarro y Clark 1998).

Considerando estas definiciones se tomó la decisión de crear un instrumento que midió tres variables a saber: comunicación con la escuela, apoyo y supervisión del aprendizaje en el hogar y conocimiento de la escuela. Para la recolección de datos sobre las variables seleccionadas se confeccionó un instrumento con escala tipo Likert, tomando como base la propuesta de Valdés et al. (Valdés et al. 2013). Se redactaron 32 ítems de los cuales se establecieron tres categorías que corresponden a 12 preguntas para la variable comunicación con la escuela, 12 preguntas para la variable apoyo y supervisión del aprendizaje en el hogar y 8 preguntas para la variable conocimiento de la escuela.

Los reactivos se redactaron en forma de afirmación en donde los Padres y Apoderados respondieron con la frecuencia con la que realizan la acción descrita, teniendo como posibles respuestas: Nunca, Pocas Veces, Algunas Veces y Siempre (ver tabla 1). 
Tabla 1. Ejemplos de Reactivos

\begin{tabular}{|l|l|l|l|l|}
\hline & Nunca & $\begin{array}{c}\text { Pocas } \\
\text { veces }\end{array}$ & $\begin{array}{c}\text { Algunas } \\
\text { veces }\end{array}$ & Siempre \\
\hline $\begin{array}{l}\text { 1. Habla continuamente con el profesor sobre los } \\
\text { resultados de aprendizajes de su hijo. }\end{array}$ & & & & \\
\hline $\begin{array}{l}\text { 2. Habla continuamente con el profesor sobre la } \\
\text { conducta de su hijo. }\end{array}$ & & & & \\
\hline $\begin{array}{l}\text { 22. Su hijo desarrolla las tareas de manera } \\
\text { independiente. }\end{array}$ & & & & \\
\hline $\begin{array}{l}\text { 23. Acompaña constantemente a si hijo en los } \\
\text { momentos en que estudia o desarrolla su tarea. }\end{array}$ & & & & \\
\hline $\begin{array}{l}\text { 29. Ha leído el manual de convivencia escolar de la } \\
\text { escuela. }\end{array}$ & & & & \\
\hline $\begin{array}{l}\text { 30. Conoce y revisa los programas y proyectos de la } \\
\text { escuela PME y PIE (si existe). }\end{array}$ & & & & \\
\hline
\end{tabular}

Fuente: Elaboración propia.

\section{ANÁLISIS DE LOS DATOS}

La confiabilidad total del instrumento se calculó según Alfa de Cronbach por medio del programa SPSS Statistic 21para IOS/MAC.

Tabla 2. Estadísticos de Confiabilidad

\begin{tabular}{|c|c|c|}
\hline Alfa de Cronbach & Alfa de Cronbach basada en los elementos tipificados & $\mathrm{N}^{\mathrm{o}}$ de elementos \\
\hline 0,901 & 0,906 & 32 \\
\hline
\end{tabular}

Fuente: Elaboración propia.

El índice de confiablidad obtenido refleja que el test se apega en 0,901 a la realidad investigada, acercándose altamente a la realidad observada, por lo que el reflejo de la coherencia interna se califica como alta considerando una coherencia total de 1,00 o $100 \%$. Obteniendo una precisión de excelencia en la medición por medio de la aplicación del instrumento (Caro, Escalante y Barahona 2002; Kerlinger y Lee 2002; Nunnally 1973, 1987; Pizarro 2012). El alcance dado por el valor alfa de 0,901, otorga un acercamiento sobre el $90 \%$ de representación de la realidad estudiada y el valor de constructo medio por medio del cuestionario creado. 
Se computó un Análisis Factorial Comprobatorio (AFc) para asociar las teorías supuestas y autores seleccionados con las respuestas al cuestionario (validez constructiva). El AFc tomó como fundamento el análisis de los componentes principales, rotación Varimax y estandarización Kayser.

Al realizar una correlación de los ítems, las agrupaciones de los factores presentaron correlaciones de variados valores, presentando valores negativos $(-0,004$ y $-0,009)$ у positivos siendo 0,551 la mayor correlación. Este Análisis Factorial Comprobatorio (AFc) muestra un valor Determinante $\mathrm{a}=2,57 \mathrm{E}-006$ acorde a las correlaciones encontradas. Otro requisito de ajuste de los datos al Análisis Factorial Comprobatorio $(\mathrm{AFc})$ corresponde al test KMO y su prueba de esfericidad de Barlett (ver Tabla 3).

Tabla 3. KMO y prueba de Bartlett

\begin{tabular}{|c|c|c|}
\hline Medida de adecuación muestral de Kaiser-Meyer-Olkin. & 0,870 \\
\hline \multirow{3}{*}{ Prueba de esfericidad de Bartlett } & Chi-cuadradoaproximado & 6532,728 \\
\cline { 2 - 3 } & Gl & 496 \\
\cline { 2 - 3 } & Sig. & 0,000 \\
\hline
\end{tabular}

Fuente: Elaboración propia.

El test KMO $(0,870)$ está excelente, pues su índice se acerca al máximo valor 1 , indicando una excelente medida de adecuación muestral. Interpretación similar a un coeficiente de correlación binario mayor y cercano a 1 , considerándose como muy aceptable al ser mayor a 0,75 .

En la prueba de Bartlett $\left(\mathrm{X}^{2}=6532,728\right)$, la probabilidad asociada, sig. $=0,000$ confirma la hipótesis de ajuste de los datos al AFc planteada a un nivel de significación del 5\%, respaldando la realización de los análisis de los factores (Caro et al. 2002; Kerlinger y Lee 2002; Nunnally 1973, 1987; Pizarro 2012).

La adecuación muestral $\mathrm{KMO}=0,870$ se considera muy apropiado para el modelo de análisis factorial realizado, considerando la amplia interrelación entre las tres variables planteadas y la cantidad de ítems del cuestionario (Caro et al. 2002; Kerlinger y Lee 2002; Nunnally 1973, 1987; Pizarro 2012). El valor KMO=0,870 para la matriz de datos es clasificado como muy meritorio para ejecutar el análisis factorial (Caro et al. 2002; Pizarro y Clark 2007; Vivanco 2005).

La aplicación de extracción de los componentes principales muestra las comunalidades iniciales totales igual a 1.0 y los valores de la extracción no se acercan a cero (ver Tabla 4). Los resultados ordenados de mayor a menor comunalidad de extracción igual o superior a 0,600 en los ítems 1- 2- 27. Entre 0,500 y 0,599 los ítems 4- 9- 10- 21- 25- 32. Entre 0,400 y 0,499 los ítems 5- 15- 16- 17- 19- 23- 26- 28- 30. Entre 0,300 y 0,399 los ítems 6- 8- 11 12- 13-14- 18- 20- 29. Entre 0,200 y 0,299 los ítems 3- 24- 31. Entre 0,100 y 0,199 los ítems 7 y 22 . No se observan valores menores a 0,100 ni cercanos a 0 siendo el más bajo el ítem $7=0,116$. 
Estudios Pedagógicos XLIV, ${ }^{\circ}$ 1: 115-133, 2018 ESTUDIO METODOLÓGICO DE LAS VARIABLES QUE COMPONEN EL CONSTRUCTO DE "APOYO Y PARTICIPACIÓN DE LA FAMILIA EN LOS PROCESOS EDUCATIVOS"

Tabla 4. Comunalidades ${ }^{1}$

\begin{tabular}{|c|c|c|}
\hline & Inicial & Extracción \\
\hline 1 & 1,000 & 0,621 \\
\hline 2 & 1,000 & 0,648 \\
\hline 3 & 1,000 & 0,206 \\
\hline 4 & 1,000 & 0,557 \\
\hline 5 & 1,000 & 0,420 \\
\hline 6 & 1,000 & 0,319 \\
\hline 7 & 1,000 & 0,116 \\
\hline 8 & 1,000 & 0,336 \\
\hline 9 & 1,000 & 0,508 \\
\hline 10 & 1,000 & 0,565 \\
\hline 11 & 1,000 & 0,334 \\
\hline 12 & 1,000 & 0,358 \\
\hline 13 & 1,000 & 0,354 \\
\hline 14 & 1,000 & 0,396 \\
\hline 15 & 1,000 & 0,439 \\
\hline 16 & 1,000 & 0,436 \\
\hline 17 & 1,000 & 0,432 \\
\hline 18 & 1,000 & 0,343 \\
\hline 19 & 1,000 & 0,410 \\
\hline 20 & 1,000 & 0,388 \\
\hline 21 & 1,000 & 0,508 \\
\hline 22 & 1,000 & 0,188 \\
\hline 23 & 1,000 & 0,425 \\
\hline 24 & 1,000 & 0,227 \\
\hline 25 & 1,000 & 0,519 \\
\hline 26 & 1,000 & 0,474 \\
\hline 27 & 1,000 & 0,617 \\
\hline 28 & 1,000 & 0,402 \\
\hline 29 & 1,000 & 0,359 \\
\hline 30 & 1,000 & 0,473 \\
\hline 31 & 1,000 & 0,275 \\
\hline 32 & 1,000 & 0,541 \\
\hline
\end{tabular}

Fuente: Elaboración propia.

\footnotetext{
Método de extracción: Análisis de Componentes principales
} 


\subsection{VARIABLES REDUNDANTES Y NO REDUNDANTES}

Los residuos se calcularon entre las correlaciones observadas y las reproducidas, existe un $62,0 \%$ de residuales redundantes con valores absolutos menores que 0,05 , existiendo una semejanza entre las correlaciones reales con las reproducidas o teóricas obtenidas en la matriz de correlaciones (Brown 2006; Pérez 2001; Pizarro 2005; Pizarro y Clark 2007; Vivanco 1999; Yela 1998). Cabe señalar un 100\% indica que los factores compuestos matemáticamente por el AFc serían idénticos a los puntajes observados según Pizarro (Pizarro 2007). Considerando un valor óptimo de 65\%, señalado por Vivanco (Vivanco 1999), el $62.0 \%$ obtenido se ajustó a lo esperado.

A su vez, la Varianza Total Explicada muestra los principales componentes del cuestionario con relación al peso de los factores revisados por medio de los autovalores iniciales. La suma total acumulada de los 3 factores de las sustracciones del cuadrado de la extracción fue de $41,23 \%$ por lo común fluctúan entre $10 \%$ - 60\%, ya que si fueran $100 \%$ los conceptos serian igual a las variables (validez y confiabilidad perfectas) (ver tabla 5).

Tabla 5. Varianza Total Explicada ${ }^{2}$

\begin{tabular}{|c|c|c|c|c|c|c|c|c|c|}
\hline \multirow{2}{*}{ Componente } & \multicolumn{3}{|c|}{ Autovalores iniciales } & \multicolumn{3}{|c|}{$\begin{array}{c}\text { Sumas de las saturaciones } \\
\text { al cuadrado de la extracción }\end{array}$} & \multicolumn{2}{|c|}{$\begin{array}{c}\text { Suma de las saturaciones al } \\
\text { cuadrado de la rotación }\end{array}$} \\
\cline { 2 - 11 } & Total & $\begin{array}{c}\% \text { de la } \\
\text { varianza }\end{array}$ & $\begin{array}{c}\% \\
\text { acumulado }\end{array}$ & Total & $\begin{array}{c}\% \text { de la } \\
\text { varianza }\end{array}$ & $\begin{array}{c}\% \\
\text { acumulado }\end{array}$ & $\begin{array}{c}\text { Total } \\
\text { \% de la } \\
\text { varianza }\end{array}$ & $\begin{array}{c}\% \\
\text { acumulado }\end{array}$ \\
\hline 1 & 8,484 & 26,514 & 26,514 & 8,484 & 26,514 & 26,514 & 4,860 & 15,188 & 15,188 \\
\hline 2 & 2,789 & 8,716 & 35,230 & 2,789 & 8,716 & 35,230 & 4,326 & 13,519 & 28,707 \\
\hline 3 & 1,921 & 6,002 & 41,231 & 1,921 & 6,002 & 41,231 & 4,008 & 12,525 & 41,231 \\
\hline 4 & 1,528 & 4,774 & 46,006 & & & & & & \\
\hline 5 & 1,347 & 4,211 & 50,217 & & & & & & \\
\hline 6 & 1,156 & 3,613 & 53,830 & & & & & & \\
\hline 7 & 1,103 & 3,448 & 57,278 & & & & & & \\
\hline 8 & 1,014 & 3,169 & 60,447 & & & & & & \\
\hline 9 & 0,919 & 2,872 & 63,319 & & & & & & \\
\hline 10 & 0,880 & 2,751 & 66,071 & & & & & & \\
\hline 11 & 0,869 & 2,717 & 68,787 & & & & & & \\
\hline 12 & 0,829 & 2,591 & 71,378 & & & & & & \\
\hline 13 & 0,767 & 2,398 & 73,777 & & & & & & \\
\hline 14 & 0,690 & 2,155 & 75,931 & & & & & & \\
\hline
\end{tabular}

Método de extracción: Análisis de Componentes principales. 


\begin{tabular}{|l|l|l|l|l|l|l|l|l|l|}
\hline 15 & 0,662 & 2,068 & 78,000 & & & & & & \\
\hline 16 & 0,633 & 1,977 & 79,977 & & & & & & \\
\hline 17 & 0,603 & 1,885 & 81,862 & & & & & & \\
\hline 18 & 0,574 & 1,793 & 83,655 & & & & & & \\
\hline 19 & 0,566 & 1,768 & 85,423 & & & & & & \\
\hline 20 & 0,515 & 1,610 & 87,033 & & & & & & \\
\hline 21 & 0,494 & 1,544 & 88,577 & & & & & & \\
\hline 22 & 0,491 & 1,534 & 90,111 & & & & & & \\
\hline 23 & 0,457 & 1,427 & 91,538 & & & & & & \\
\hline 24 & 0,424 & 1,326 & 92,863 & & & & & & \\
\hline 25 & 0,397 & 1,241 & 94,104 & & & & & & \\
\hline 26 & 0,343 & 1,073 & 95,177 & & & & & & \\
\hline 27 & 0,326 & 1,020 & 96,197 & & & & & & \\
\hline 28 & 0,306 & 0,955 & 97,152 & & & & & & \\
\hline 29 & 0,260 & 0,812 & 97,964 & & & & & & \\
\hline 30 & 0,247 & 0,772 & 98,736 & & & & & & \\
\hline 31 & 0,237 & 0,741 & 99,477 & & & & & & \\
\hline 32 & 0,167 & 0,523 & 100,00 & & & & & & \\
\hline & & & & & & & \\
\hline 29
\end{tabular}

La observación de la matriz de varianza total explicada, muestra que los tres componentes explican un total de $41,231 \%$ del total de la varianza para las saturaciones al cuadrado de la extracción, siendo el componente $1=26,514 \%$, componente $2=8,716$ y componente $3=6,002 \%$.

La saturación al cuadrado de la rotación muestra que los tres componentes explican el $41,231 \%$ de la varianza, teniendo variaciones en los pesos por factor, componente $1=8,48$. Componente $2=2,79$. Componente $3=1,92$ el porcentaje de varianza explicada se puede apreciar visualmente en el gráfico de sedimentos en la figura 1. 
Figura 1: Gráfico de Sedimentación

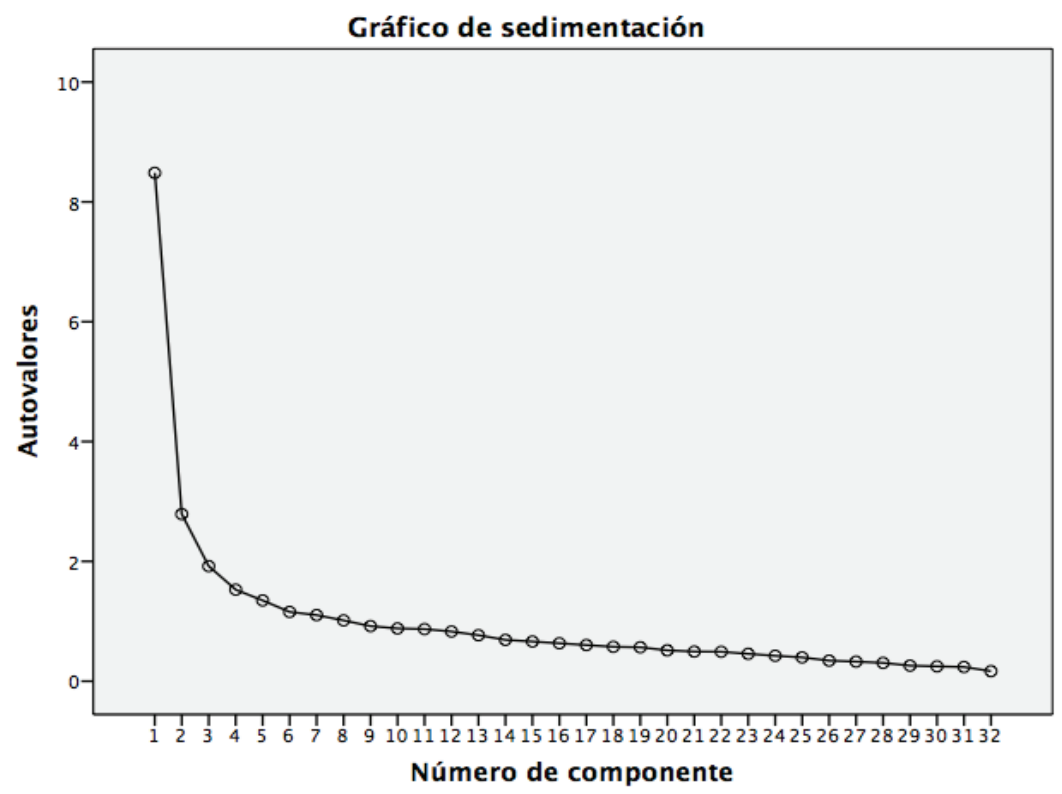

El gráfico de sedimentos en donde "van cayendo de la roca las arenillas de menor o poco peso", muestra los valores del total de la suma de las saturaciones al cuadrado de la extracción, coincidiendo los valores con los de los autovalores iniciales y en donde los tres primeros componentes explican el 41,231\%: 8,484 para el primer componente, 2,789 para el segundo componente y 1,921 para el tercer componente.

Se realizó un análisis de componentes $(\mathrm{AFc})$ para determinar la pureza de los ítems en relación con el cuestionario completo usando peso factorial a partir de la rotación de los componentes (ver Tabla 6):

Tabla 6. Matriz de componentes rotados ${ }^{3}$

\begin{tabular}{|c|c|c|c|}
\hline \multirow{2}{*}{} & \multicolumn{3}{|c|}{ Componente } \\
\cline { 2 - 4 } & 1 & 2 & 3 \\
\hline 1 & 0,759 & 0,169 & 0,128 \\
\hline 2 & 0,785 & 0,111 & 0,142 \\
\hline 3 & 0,431 & 0,115 & \\
\hline
\end{tabular}

Método de extracción: Análisis de componentes principales.

Método de rotación: Normalización Varimax con Kaiser: La rotación ha convergido en 5 iteraciones 
Estudios Pedagógicos XLIV, ${ }^{\circ}$ 1: 115-133, 2018 ESTUDIO METODOLÓGICO DE LAS VARIABLES QUE COMPONEN EL CONSTRUCTO DE "APOYO Y PARTICIPACIÓN DE LA FAMILIA EN LOS PROCESOS EDUCATIVOS"

\begin{tabular}{|c|c|c|c|}
\hline 4 & 0,732 & & 0,141 \\
\hline 5 & 0,628 & & 0,160 \\
\hline 6 & 0,494 & 0,241 & 0,128 \\
\hline 7 & 0,207 & 0,237 & 0,128 \\
\hline 8 & 0,451 & 0,338 & 0,137 \\
\hline 9 & 0,710 & & \\
\hline 10 & 0,736 & & 0,147 \\
\hline 11 & 0,459 & 0,107 & 0,334 \\
\hline 12 & 0,344 & 0,324 & 0,367 \\
\hline 13 & & 0,591 & \\
\hline 14 & & 0,606 & $-0,155$ \\
\hline 15 & & 0,657 & \\
\hline 16 & 0,198 & 0,604 & 0,179 \\
\hline 17 & 0,166 & 0,563 & 0,295 \\
\hline 18 & 0,300 & 0,450 & 0,225 \\
\hline 19 & 0,269 & 0,515 & 0,270 \\
\hline 20 & & 0,576 & 0,224 \\
\hline 21 & & 0,694 & 0,132 \\
\hline 22 & & 0,382 & 0,205 \\
\hline 23 & 0,231 & 0,446 & 0,416 \\
\hline 24 & & 0,419 & 0,209 \\
\hline 25 & 0,373 & 0,178 & 0,590 \\
\hline 26 & 0,102 & 0,136 & 0,667 \\
\hline 27 & 0,182 & 0,142 & 0,751 \\
\hline 28 & & 0,367 & 0,510 \\
\hline 29 & 0,241 & 0,133 & 0,533 \\
\hline 30 & 0,261 & & 0,636 \\
\hline 31 & & & 0,518 \\
\hline 32 & 0,223 & 0,247 & 0,656 \\
\hline
\end{tabular}

Fuente: Elaboración propia. 
La rotación de los componentes muestra que los ítems usando peso factorial 0,10 por default del programa SPSS Statistic 21, en su mayoría poseen cargas que pertenecen a los tres componentes. Si es posible observar por el peso factorial de los componentes, los ítems miden mayormente a cada uno según el orden que se dio a los ítems en el cuestionario.

Para el componente 1 los ítems del 1 al 12 poseen mayor carga factorial, siendo el ítem $2=0,785$ la mayor carga y el ítem $7=0,207$ la menor carga factorial. También se encuentra el ítem 12 como un ítem que posee una carga factorial estadísticamente igual en los tres componentes y el ítem $9=0,710$ se observa como ítem puro.

Para el componente 2 los ítems del 13 al 24 poseen mayor carga factorial, en donde el ítem $21=0,694$ es el valor más alto y el ítem $22=0,382$ es el valor más bajo. Los ítems puros son $13=0,591$ e ítem $15=0,694$ ambos para el factor 2 .

En el componente 3 los ítems del 25 al 32 poseen mayor carga factorial y se observa al ítem $27=0,751$ como la mayor carga factorial. Y, al ítem $28=0,510$ como la menor carga factorial. El ítem 31 = 0,518 se observa como el único ítem puro para este componente.

\subsubsection{Correlaciones inter factoriales}

La influencia de las variables (comescuela, apoyohogar y conescuela) que componen la participación familiar en los procesos educativos (total) mantienen las siguientes relaciones (ver Tablas 7 y 8 ):

Tabla 7. Estadísticos Descriptivos de las Variables (N=520).

\begin{tabular}{|l|c|c|}
\hline & Media & Desviación típica \\
\hline Total & 109,98 & 12,918 \\
\hline comescuela & 40,81 & 6,351 \\
\hline apoyohogar & 44,25 & 4,132 \\
\hline conescuela & 24,92 & 5,307 \\
\hline
\end{tabular}

Fuente: Elaboración propia

La descripción de los puntajes alcanzados en el test total y seccionada por cada una de las variables refleja que la mayor media aritmética corresponde a la variable apoyohogar (apoyo y supervisión del aprendizaje en el hogar), obteniendo una media aritmética de 44,25 . Y una desviación típica $=4,132$ puntos, considerada baja para un total ideal de puntaje $=48$. comescuela (comunicación con la escuela) con una media aritmética de 40,81 y una desviación típica $=6,351$, la más amplia de los tres factores, pero considerada baja para un puntaje total $=48$. Finalmente, conescuela (conocimiento de la escuela) $\operatorname{con} 24,92$ puntos y desviación típica $=5,307$, puntaje bajo para un puntaje total $=32$. A su vez las correlaciones entre el constructo y cada una de las variables se expresa de manera positiva, explicándose la participación de la familia en los procesos educativos ordenadamente de mayor a menor por la relación los puntajes obtenidos (ver Tabla 6): 
Tabla 8. Correlaciones inter variables.

\begin{tabular}{|c|c|c|c|c|c|}
\hline & & total & comescuela & apoyohogar & conescuela \\
\hline \multirow[t]{3}{*}{ Total } & Correlación de Pearson & 1 & $0,848^{* *}$ & $0,756^{* *}$ & $0,831^{* *}$ \\
\hline & Sig. (bilateral) & & 0,000 & 0,000 & 0,000 \\
\hline & $\mathrm{N}$ & 520 & 520 & 520 & 520 \\
\hline \multirow[t]{3}{*}{ comescuela } & Correlación de Pearson & $0,848^{* *}$ & 1 & $0,453^{* *}$ & $0,516^{* *}$ \\
\hline & Sig. (bilateral) & 0,000 & & 0,000 & 0,000 \\
\hline & $\mathrm{N}$ & 520 & 520 & 520 & 520 \\
\hline \multirow[t]{3}{*}{ apoyohogar } & Correlación de Pearson & $0,756^{* *}$ & $0,453^{* *}$ & 1 & $0,520^{* *}$ \\
\hline & Sig. (bilateral) & 0,000 & 0,000 & & 0,000 \\
\hline & $\mathrm{N}$ & 520 & 520 & 520 & 520 \\
\hline \multirow[t]{3}{*}{ conescuela } & Correlación de Pearson & $0,831^{* *}$ & $0,516^{* *}$ & $0,520^{* *}$ & 1 \\
\hline & Sig. (bilateral) & 0,000 & 0,000 & 0,000 & \\
\hline & $\mathrm{N}$ & 520 & 520 & 520 & 520 \\
\hline
\end{tabular}

Fuente: Elaboración propia.

El índice o coeficiente de correlación se calculó por medio del SPSS obteniendo resultados para Correlación de Pearson y elevando sustantivamente los resultados al cuadrado para determinar el \% de comunalidad en relación a la varianza obtenida.

El $\mathrm{r}$ para total/comescuela $=0,848(\mathrm{p}<0,01)$, compartiendo un total de $71,91 \%$ de la varianza. El $\mathrm{r}$ total/conescuela $=0,831$, compartiendo un $69.05 \%$ de la varianza total $(\mathrm{p}<0,01)$. Y el $\mathrm{r}$ total/apoyohogar $=0,756$ que demuestra que los puntajes comparten un $57,15 \%$ de la varianza $(\mathrm{p}<0,01)$, lo que demuestra altas, significativas y reales relaciones entre el constructo y las variables que lo componen.

La variable comescuela posee correlaciones positivas con las demás variables, siendo $\mathrm{r}$ comescuela/apoyohogar $=0,453(\mathrm{p}<0,01) . \mathrm{r}$ comescuela/ conescuela $=0,516(\mathrm{p}<$ $0,01)$. De esta manera realizado el cálculo de para obtener las comunalidades, se obtuvo como resultado que comescuela/ apoyohogar comparten un $20,05 \%$ de la varianza y que comescuela/ conescuela comparten un $26,62 \%$ de la varianza.

Apoyohogar refleja correlaciones positivas en $\mathrm{r}$ apoyohogar/ comescuela $=0,453(\mathrm{p}<$ $0,01)$ y $\mathrm{r}$ apoyohogar/ conescuela $=0,520(\mathrm{p}<0,01)$. De esta manera la representación de las comunalidades obtenidas fueron $20,05 \%$ y $27,04 \%$.

De la misma manera $\mathrm{r}$ conescuela/ comescuela $=0,516(\mathrm{p}<0,01)$ y $\mathrm{r}$ conescuela/ apoyohogar $=0,520(\mathrm{p}<0,01)$. Por lo que se puede asegurar que las correlaciones descritas comparten $26,62 \%$ y $27,04 \%$ de la varianza respectivamente. 
Estudios Pedagógicos XLIV, N $^{\circ}$ 1: 115-133, 2018

ESTUDIO METODOLÓGICO DE LAS VARIABLES QUE COMPONEN EL CONSTRUCTO DE "APOYO Y PARTICIPACIÓN DE LA FAMILIA EN LOS PROCESOS EDUCATIVOS"

\section{CONCLUSIONES Y REFLEXIONES FINALES}

Un cálculo entre las correlaciones observadas y reproducidas que dio como resultado un $62,0 \%$ de residuos redundantes que poseen valores absolutos menores que 0,05 . Ello refleja la existencia de similitudes entre las correlaciones reales con las reproducidas o teoricamente obtenidas en la matriz. Considerando que lo óptimo es obtener un $65 \%$ $(\mathrm{p}<0,05)$ de residuales redundantes, el $62 \%(\mathrm{p}<0,05)$ se ajusta a lo esperado e indica que los componentes matemáticos se apegan a los puntajes observados. Así se confirma la hipótesis, ya que el $62 \%(\mathrm{p}<0,05)$ obtenido se ajusta a lo esperado según las propuestas teóricas descritas por Brown; Pérez; Yela; Vivanco; Pizarro; Pizarro y Clark (Brown 2006; Pérez 2001; Yela 1998; Vivanco 1999; Pizarro 2005, 2006; Pizarro y Clark 1998, 2007).

"Comprobar por medio de $\mathrm{AF}(\mathrm{c})$ la influencia de las variables: Comunicación con la Escuela, Apoyo y Supervisión del Aprendizaje en el Hogar y Conocimiento de la Escuela en el constructo de Participación y Apoyo Familiar en los procesos educativos", se comprueba que las variables/ componentes poseen una influencia alta en el constructo, esto comprobado por medio de los residuos redundantes $=62,0 \%$ con valores absolutos menores que 0,05 . mostrando la existencia de similitudes entre las correlaciones reales con las reproducidas o teoricamente obtenidas en la matriz.

También al observar las correlaciones entre las variables y el constructo se observa que el constructo Participación y Apoyo Familiar en los procesos educativos posee correlaciones positivas significativas con las tres variables que lo componen. El componente Comunicación con la Escuela posee la correlación más alta $\mathrm{r}=0,848$. El componente Conocimiento de la Escuela, posee la segunda correlación más alta r=0,831. Y finalmente, el componente Apoyo y Supervisión del Aprendizaje en el Hogar se refleja en una correlación r=0,756. Teniendo todas, una significancia bilateral menor que 0,01 . Por lo que se considera que las variables seleccionadas y planteadas desde los modelos teóricos usados para el planteamiento del constructo Participación y Apoyo Familiar en los Procesos Educativos. Es decir, poseen una alta influencia sobre el mismo, teniendo un orden lógico en donde la existencia de una variable, otorga un sustento para la existencia de las demás variables.

La búsqueda de "Determinar el "peso" de las variables descritas en el constructo de participación de la Familia en los procesos educativos", se realizó por medio del cálculo de correlaciones entre los componentes y el constructo, en donde: la correlación de la variable apoyohogar con el constructo total es $=0,756$ significativa a nivel de 0,01 . Es necesario destacar que las variables comescuela y conescuela poseen un peso mayor, siendo $r=0,848$ y $\mathrm{r}=0,831$ los valores respectivamente. A partir de estos datos obtenidos es que el orden de las variables, determinado por el valor $\mathrm{r}$ alcanzado es el que otorga un orden a los procesos en donde influyen estas variables, la que elevada al cuadrado explica el \% de representación de la varianza total del constructo. La Comunicación con la Escuela (comescuela), variable que representa un $71,91 \%$ de la varianza total; seguido de Conocimiento de la Escuela (conescuela), que alcanza un 69,05\% del total de la varianza; El Apoyo y supervisión del Aprendizaje en el Hogar (apoyohogar), representando un 57,15\% de la varianza.

Efectuado este análisis, se puede afirmar que la Participación Familiar en los procesos educativos posee un orden natural de ejecución basado en los pesos de las variables que la componen y ordenan este fenómeno. Por lo que al existir comunicación con la escuela (comescuela r=0,848) permite el conocimiento de la escuela (conescuela $\mathrm{r}=0,831$ ) permitiendo el desarrollar Apoyo y Supervisión del Aprendizaje en el Hogar (apoyohogar r=0,756). 


\section{REFERENCIAS BIBLIOGRÁFICAS}

Álvarez, B., Aguirre, Mª y Vaca, S. (2010). Interacción familia-escuela: Análisis de contenido sobre el discurso de docentes y familias. Revista Española de Orientación y Psicopedagogía, 21 (2), 320-334. doi: 10.5944/reop.vol.21.num.2.2010.11535.

Anabalón, M., Carrasco, S., Díaz, D., Gallardo, C. y Cárcamo, H. (2008). El compromiso familiar frente al desempeño escolar de niños y niñas de educación general básica en la ciudad de Chillán. Revista Horizontes Educacionales, 13 (1), 11-21. Recuperado de http://www.redalyc. org/articulo.oa?id=97912446001.

Arancibia, V. (1992). Efectividad escolar: Un análisis comparado. Estudios Públicos, (47), 101-125. Recuperado de https://www.cepchile.cl/cep/site/artic/20160303/asocfile/20160303184840/ rev47_arancibia.pdf.

Bazán, A., Sánchez, B. y Castañeda, S. (2007). Relación estructural entre el apoyo familiar, nivel educativo de los padres, características del maestro y desempeño en la lengua escrita. Revista mexicana de investigación educativa, 12 (33), 701-729. Recuperado de http://www.redalyc.org/ articulo.oa?id=14003312.

Bellei, C., Morawietz, L., Valenzuela, J. y Vanni, X. (2015). Nadie dijo que era fácil. Escuelas efectivas en sectores de pobreza, diez años después. Santiago: LOM ediciones.

Bellei, C., Valenzuela, J., Vanni, X. y Contreras, D. (2014). Lo aprendí en la escuela: ¿cómo se logran procesos de mejoramiento escolar?. Santiago: Universidad de Chile.

Belmar, B. (2003). Familia y escuela: realidades y desafíos. Revista Horizontes Educacionales, 8, 7-14. Recuperado de http://www.redalyc.org/articulo.oa?id=97917166002.

Bolívar, A. (2006). Familia y escuela: dos mundos llamados a trabajar en común. Revista de Educación, 339, 119-146. Recuperado de http://ibdigital.uib.es/greenstone/collect/portal_social/ index/assoc/miso1089/4_002.dir/miso10894_002.pdf.

Brown, A. (2006). Comfirmatory factor Analysis. New York: The Guilford Press.

Brunner, J. (2003). Informe de capital humano en Chile. Santiago: Universidad Adolfo Ibáñez.

Brunner, J y Elacqua, G. (2004). Factores que inciden en la educación efectiva. Evidencia internacional. La Educación, (139-140), 1-11. Recuperado de https://www.educoas.org/portal/ bdigital/lae-ducacion/

Cano, R. y Casado, M. (2015). Escuela y familia: Dos pilares fundamentales para unas buenas prácticas de orientación educativa a través de las escuelas para padres. Revista Electrónica Interuniversitaria de Formación del Profesorado, 18 (2), 15-28. doi: 10.6018/reifop.18.2.219491.

Caro, A., Escalante, E., y Barahona, L. (2002). Análisis y Tratamiento de datos en SPSS. Valparaíso: Ediciones Universidad de Playa Ancha.

Coraggio, J. L. (1997). Las propuestas del Banco Mundial para la educación: ¿sentido oculto o problemas de concepción?. En J.L. Coraggio y R.M. Torres, La educación según el Banco Mundial. Un análisis de sus propuestas y métodos (pp. 1-45). Buenos Aires: Miño y DávilaCentro Estudios Multidisciplinarios. Recuperado de https://pdfs.semanticscholar.org/0aa4/ b5ce767410593b861f3e6b3769e9e20b749f.pdf

Cornejo, R. y Redondo, J. (2007). Variables y factores asociados el aprendizaje escolar: una discusión desde la investigación actual. Revista Estudios Pedagógicos, 33 (2), 155-175. doi: 10.4067/ S0718-07052007000200009.

Epstein, J. y Sheldon, S. (2008). Moving forward: Ideas for research on school, family, an community partnerships. En C. Chifton y S. Ronald (Eds), Handbook for research in education (pp.115150). California: Sage Publications.

Fullan, M. (2011). Investigación sobre cambio educativo: presente y futuro. Revista digital de investigación Lasaliana, (3), 31-35. Recuperado de https://dialnet.unirioja.es/descarga/ articulo/3886329.pdf

Gerstenfeld, P. (1995). Comparación regional del impacto de las características del hogar en el logro 
Estudios Pedagógicos XLIV, $\mathrm{N}^{\circ}$ 1: 115-133, 2018

ESTUDIO METODOLÓGICO DE LAS VARIABLES QUE COMPONEN EL CONSTRUCTO DE “APOYO Y PARTICIPACIÓN DE LA FAMILIA EN LOS PROCESOS EDUCATIVOS"

escolar. En Naciones Unidas, Serie Políticas Sociales. Variables extrapedagógicas y equidad en la educación media: hogar, subjetividad y cultura escolar (pp. 13-37). Santiago: CEPAL.

Gomila, Ma . y Pascual, B. (2015). La participación de las familias en el sistema educativo: la percepción del profesorado en formación. Revista Electrónica Interuniversitaria de Formación del Profesorado, 18 (3), 99-112. doi: 10.6018/reifop.18.3.199321.

Himmel, E., Maltes, S. y Majluf, N. (1984). Análisis de la influencia de factores alterables del proceso educativo sobre la efectividad escolar. Santiago: Universidad Católica de Chile.

Jadue, G. (2003). Transformaciones familiares en Chile: riesgo creciente para el desarrollo emocional, psicosocial y la educación de los hijos. Revista Estudios Pedagógicos, (29), 115-126. doi: 10.4067/S0718-07052003000100008.

Jadue, G. (1999). Hacia una mayor permanencia en el sistema escolar de los niños en riesgo de bajo rendimiento y deserción. Revista Estudios Pedagógicos, (25), 83-90. doi: 10.4067/S071807051999000100005.

Kerlinger, E. y Lee, B. (2002). Investigación del Comportamiento. Métodos de Investigación en ciencias sociales. México: McGraw-Hill.

LLECE/UNESCO. (2002). Estudio cualitativo en escuelas latinoamericanas con resultados destacables en siete países latinoamericanos. Santiago: UNESCO.

Murillo, F. (Coord.) (2007). Investigación Iberoamericana sobre Eficacia Escolar. Bogotá: Convenio Andrés Bello.

Nunnally, J. (1987). Teoría psicométrica. México: Trillas.

Nunnally, J. (1973). Introducción a la medición psicológica. Buenos Aires: Paidós.

Pérez, C. (2001). Técnicas estadísticas con SPSS. Madrid: Prentice Hall.

Pizarro, R. (2012). Lenguajes investigativos educacionales: supuestos, comparaciones, síntesis. Valparaíso: Universidad de Playa Ancha.

Pizarro, R. (2009). Índices de Riesgos Educativos (IREs). Viña del Mar: EV Síntesis y Excelencias Educativas.

Pizarro, R. (2008). Sistemas educativos formales y efectos Mateo, Regresivo y Robin Hood. Boletín de Investigación Educacional, 23 (2). 13-38. Santiago: Pontifica Universidad Católica de Chile.

Pizarro, R. (2006). Anexo $N^{o} 4$ Proyecto FONDECYT N ${ }^{o} 1040251$. Santiago: FONDECYT-CONICYT.

Pizarro, R. (2005). Inteligencias múltiples, currículum del hogar, intereses, autoestimas y logros académicos previos y actuales. Revista de Psicología, 14 (2), 163-176. Recuperado de www. revistas.uchile.cl/index.php/RDP/article/viewFile/17430/18188.

Pizarro, R. y Clark, S. (2007). Static and dynamic influences of multiple intelligences, curriculum of the home, interest, self-esteems, previous learning factor on current learning. $88^{\circ}$ Annual Conference of The American Educational Research Association (AERA-SIG: multiple intelligences: Theory and Practice), April 9-14, Chicago: Proyecto FONDECYT n ${ }^{\circ} 1040251$, Uplaced, 2003.

Pizarro, R. y Clark, S. (1998). Currículum del hogar y aprendizajes: Interacción versus status. Revista de Psicología de la Universidad de Chile, 7, 25-34. Recuperado de http://www.revistapsicologia. uchile.cl/index.php/RDP/article/view/18764.

Pizarro, R. y Clark, S. (1987). El ambiente educativo del hogar. Diálogos Educacionales, (9-10), 66-83.

Ruíz, M. (2003). Educación de adultos y familia. En M. Bertely (coord.), Educación, derechos sociales y equidad. Tomo II. (pp. 713-743). México: COMIE-SEP-CESU.

UNESCO. (2015). Informe de resultados: Factores asociados TERCE. Santiago: UNESCO.

UNESCO. (2014). Informe de seguimiento de la Educación para Todos en el mundo. EnseñanzaAprendizaje logar calidad para todos. Paris, UNESCO.

UNESCO. (2012). Informe de la comisión sobre la educación para el siglo XXI. La Educación Encierra un Tesoro. Paris: UNESCO.

UNESCO. (2011). Informe de seguimiento de la educación para todos en el mundo. Una crisis 
encubierta: conflictos armados y educación. Paris: UNESCO.

UNESCO. (2008). Eficacia escolar y factores asociados en América Latina y el Caribe. Santiago: UNESCO.

UNESCO. (2000). Foro mundial sobre la educación. Dakar: UNESCO.

UNICEF. (2004). Escuelas efectivas en sectores de pobreza, ¿quién dijo que no se puede? Recuperado el 4 Noviembre 2016 desde http://www.unicef.cl/centrodoc/escuelas_efectivas/ escuela\%20efectivas.pdf

Valdés, C., Carlos, E. y Arreola, C. (2013). Desarrollo de un instrumento para medir la participación de los padres en la educación escolar de los hijos. Revista de Evaluación Educativa, 2 (1), 92109. Recuperado de http://revalue.mx/revista/index.php/revalue/article/view/55/117.

Valdés, A., Martín, M. y Sánchez, P. (2009). Participación de padres de alumnos en educación primaria en las actividades académicas de sus hijos. Revista Electrónica de Investigación Educativa, 11 (1), 1-17. Recuperado de https://redie.uabc.mx/redie/article/view/229/386

Vera, J., González, C. y Hernández, S. (2014). Familia y logro escolar en matemáticas del primer ciclo escolar de educación primaria en Sonora, México. Revista Estudios Pedagógicos, 40 (1), 281-292. doi: 10.4067/S0718-07052014000100017.

Villarroel, G. (2002). Relación familia y escuela: un estudio comparativo en la ruralidad. Revista Estudios Pedagógicos (28), 123-141. doi: 10.4067/S0718-07052002000100007.

Vivanco, M. (2005). Muestreo Estadístico: Diseño y Aplicaciones. Santiago: Editorial Universitaria.

Vivanco, M. (1999). Análisis Estadístico Multivariable. Teoría y Práctica. Santiago: Editorial Universitaria.

Yela, M. (1998). Análisis Factorial. Madrid: Editorial Paidós.

Yurén, M ${ }^{a}$. y Cruz, M. (2009). La relación familia-escuela: condición de mejora de la eficacia escolar en la formación valoral de niños(as) migrantes. Revista Iberoaméricana sobre Calidad, Eficacia y Cambio en Educación, 7 (2), 131-150. Recuperado de http://www.redalyc.org/ pdf/551/55111725008.pdf

Zárate, G. (1992). Experiencias educativas exitosas. Un análisis a base de testimonios. Santiago: Centro de Estudios Públicos. Documento de trabajo nº 175. 
